\title{
TRANSFORMATIONS OF ORDINARY DIFFERENTIAL EQUATIONS VIA DARBOUX TRANSFORMATION TECHNIQUE
}

\author{
N.V. USTINOV
}

\begin{abstract}
A new approach for obtaining the transformations of solutions of nonlinear ordinary differential equations representable as the compatibility condition of the overdetermined linear systems is proposed. The corresponding transformations of the solutions of the overdetermined linear systems are derived in the frameworks of the Darboux transformation technique.
\end{abstract}

\section{INTRODUCTION}

The nonlinear ordinary differential equations (ODE's) admitting a representation as the compatibility condition of the overdetermined system of linear equations (Lax pair) are intensively studied in modern mathematical physics. The most famous equations belonging to this class are the six Painlevé equations $\mathrm{P}_{\mathrm{I}}-\mathrm{P}_{\mathrm{VI}}$ 1. 2, 3. 3. It was found that these equations are closely connected with nonlinear partial differential equations (PDE's) integrable in the frameworks of the inverse scattering transformation (IST) method 佃. Various approaches developed in the theory of nonlinear integrable PDE's were applied to study ODE's of such a class (e.g., see reviews [5, 6] and references therein).

It is well known that $\mathrm{P}_{\mathrm{II}}-\mathrm{P}_{\mathrm{VI}}$ have the transformations that map the solutions of a given Painlevé equation into solutions of the same equation but with different values of the parameters of the equation. Such transformations for $\mathrm{P}_{\mathrm{II}}$ were found in $[7$ as a generalization of corresponding formulas of the rational solutions transformations 8 . The transformations of solutions of $\mathrm{P}_{\mathrm{II}}$ were also derived by means of the Bäcklund transformation technique [9]. A representation of the Painlevé equations as the systems of the first order equations was used to obtain the transformations of $\mathrm{P}_{\mathrm{III}}$ in the cases of two different choices of values of the parameters 10, 11], $\mathrm{P}_{\mathrm{IV}}$ [12] and of $\mathrm{P}_{\mathrm{V}}$ with special parameter values [1]. In similar manner the transformations for $\mathrm{P}_{\mathrm{II}}$ and $\mathrm{P}_{\mathrm{IV}}$ were obtained in [13]. Moreover, an equivalence of nonlinear ODE to a system of equations was used to construct the transformations of solutions of the higher order nonlinear ODE's appearing as the self-similar reduction in the KdV hierarchy [13, 14]. An unified approach to investigate the transformation properties of Painlevé equations, which utilizes the singularity structure of their solutions, were developed in [15]. The known transformations and new ones for $\mathrm{P}_{\mathrm{V}}$, that differs from the equation considered in 11] by a region of the parameters values, and for $\mathrm{P}_{\mathrm{VI}}$ were derived. The truncation method was modified to find the transformations of nonlinear ODE's [16]. Its extension, which is based on considering the transformations that preserve the locations of subsets of the singularities of solutions, was suggested [17]. The transformations of solutions of $\mathrm{P}_{\mathrm{II}}$ and $\mathrm{P}_{\mathrm{IV}}$ were, in particular, obtained using this approach.

Supported by RFFI grant 97-01-00752. 
The transformations of nonlinear ODE representable as the compatibility condition of the Lax pair cause evidently the transformations of the Lax pair solutions. Studying the monodromy preserving deformations of matrix linear ODE's with regular singularities, Schlesinger 18] constructed, in pure algebraic way, the transformations that keep the monodromy data of the solutions except for the exponents of formal monodromy. These transformations, which are now referred to as the Schlesinger transformations, were generalized for linear equations with irregular singular points by Jimbo and Miwa [3]. The monodromy preserving condition is equivalent to having a set of additional linear ODE's on solution of the isomonodromic equation 18, 19, which establish a hierarchy of the Lax pairs. However, the authors mentioned seem to apply no the Schlesinger transformations for the derivation of transformations for nonlinear ODE's (such as Painlevé equations; see [3, p. 437) that admit the compatibility condition representation. The transformations of $\mathrm{P}_{\mathrm{II}}-\mathrm{P}_{\mathrm{VI}}$, including new ones, were obtained by means of the Schlesinger transformations of associated Lax pairs in [20].

In the present paper we show that the Darboux transformation (DT) technique 21 can be used to construct the transformations of solutions of nonlinear ODE's and associated Lax pairs. This technique is suitable for generating the infinite hierarchies of solutions satisfying the reduction constraints imposed on the coefficients of the Lax pairs. To extend the DT technique for obtaining the transformations of ODE's we suggest that the points, in which the DT possesses a kernel, coincide with the singular points of the Lax pairs. The cases of $\mathrm{P}_{\text {II }}$ and certain nonlinear ODE of the second order are examined by way of illustration.

The paper is organized as follows. The Lax pairs of the ODE's under consideration are written in Sec.2. We present the explicit formulas of the DT's in Sec.3 and discuss the properties of them. The asymptotic expansions at the singular point neighborhood of the Lax pairs solutions are given in Sec.4. These expansions are used there to build the transformations of the ODE's on the basis of the DT formulas.

\section{LAX PAirs AND Nonlinear ODE's}

Let us consider direct Lax pair

$$
\left\{\begin{array}{l}
\Psi_{x}=P \Psi \\
\Psi_{\lambda}=Q \Psi
\end{array}\right.
$$

for matrix function $\Psi \equiv \Psi(x, \lambda)$ and dual Lax pair

$$
\left\{\begin{array}{l}
\Xi_{x}=-\Xi P \\
\Xi_{\lambda}=-\Xi Q
\end{array}\right.
$$

for matrix $\Xi \equiv \Xi(x, \lambda)$. The compatibility condition of the direct pair $\Psi_{x \lambda}=\Psi_{\lambda x}$ (or, for dual one, $\Xi_{x \lambda}=\Xi_{\lambda x}$ ) leads to the condition on the matrix coefficients $P$ and $Q$ :

$$
P_{\lambda}-Q_{x}+[P, Q]=0
$$

The equation $\mathrm{P}_{\mathrm{II}}$

$$
u_{x x}=2 u^{3}+x u-\alpha
$$

arises from the compatibility condition of Lax pairs (112) with coefficients

$$
P=-\lambda \sigma_{3}+u \sigma_{1},
$$




$$
Q=4 \lambda^{2} \sigma_{3}-4 \lambda u \sigma_{1}+\sigma_{3}\left(2 u_{x} \sigma_{1}-2 u^{2} E-x E\right)+\lambda^{-1} \alpha \sigma_{1},
$$

where $\sigma_{i}(i=1,2,3)$ are the Pauli spin matrices, $E$ is $2 * 2$ unit matrix.

In the sequel the DT technique will be applied for obtaining the transformations of the solutions of $\mathrm{P}_{\mathrm{II}}$ and following ODE

$$
x^{2} u u_{x x}=\left(x u_{x}\right)^{2}-x u u_{x}+\alpha^{2} u^{4}-2 \gamma u^{3}-2(2 \beta+1) x u-4 x^{2}
$$

( $\alpha, \beta$ and $\gamma$ are constants), for which the transformations were not written explicitly

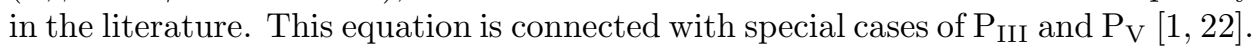

Let us consider direct and dual Lax pairs $(1,2)$ with coefficients

$$
\begin{gathered}
P=-\lambda \sigma_{3}+\frac{1}{x}\left(\begin{array}{cc}
0 & v_{1} \\
v_{2} & 0
\end{array}\right), \\
Q=-x \sigma_{3}+\lambda^{-1}\left(\begin{array}{cc}
\beta & v_{1} \\
v_{2} & -\beta
\end{array}\right)+\lambda^{-2}\left(\begin{array}{cc}
q & w_{1} \\
w_{2} & -q
\end{array}\right),
\end{gathered}
$$

where $v_{1}=u z w, v_{2}=\frac{v(\alpha-z)}{w}, w_{1}=z w, w_{2}=\frac{\alpha-z}{w}, q=z-\frac{\alpha}{2}$. The compatibility condition (3) in this case yields

$$
\left\{\begin{array}{l}
u_{x}=-2-\frac{2 \beta}{x} u+\frac{2 z-\alpha}{x} u^{2} \\
v_{x}=2+\frac{2 \beta}{x} v-\frac{2 z-\alpha}{x} v^{2} \\
z_{x}=\frac{v-u}{x} z(z-\alpha) \\
\frac{w_{x}}{w}=-\frac{u z+v(z-\alpha)}{x}
\end{array}\right.
$$

The first three equations are reduced to Eq.(7).

\section{Darboux Transformation Technique}

The first equations of Lax pairs (1,2) with coefficient $P$ given by Eq.(5) or Eq.(8) are well known in the theory of IST as direct and dual Zakharov-Shabat (ZS) spectral problems

$$
\begin{gathered}
\Psi_{x}=\left(\lambda P^{(1)}+P^{(0)}\right) \Psi, \\
\Xi_{x}=-\Xi\left(\lambda P^{(1)}+P^{(0)}\right) .
\end{gathered}
$$

Potential $P^{(0)}$ of these problems has following general form:

$$
P^{(0)}=\left(\begin{array}{cc}
0 & u_{1} \\
u_{2} & 0
\end{array}\right) .
$$

The DT technique allows one to produce new solutions of the ZS problems and corresponding matrix potential, starting from the initial ones. Let $\varphi=\left(\varphi_{1}, \varphi_{2}\right)^{T}$ be a vector solution of direct $\mathrm{ZS}$ problem $(10)$ with $\lambda=\mu$. The first elementary DT (EDT) of direct problem $\left\{\Psi, \Xi, P^{(0)}\right\} \rightarrow\left\{\tilde{\Psi}, \tilde{\Xi}, \tilde{P}^{(0)}\right\}$ is defined as given [23, 24]:

$$
\tilde{\Psi}=\sigma\left(\begin{array}{cc}
\lambda-\mu+\frac{u_{1} \varphi_{2}}{2 \varphi_{1}} & -\frac{u_{1}}{2} \\
-\frac{\varphi_{2}}{\varphi_{1}} & 1
\end{array}\right) \Psi, \quad \tilde{\Xi}=\frac{\Xi}{\sigma}\left(\begin{array}{cc}
1 & \frac{u_{1}}{2} \\
\frac{\varphi_{2}}{\varphi_{1}} & \lambda-\mu+\frac{u_{1} \varphi_{2}}{2 \varphi_{1}}
\end{array}\right)
$$




$$
\tilde{P}^{(0)}=\left(\begin{array}{cc}
0 & -\frac{u_{1, x}}{2}+\frac{u_{1}^{2} \varphi_{2}}{2 \varphi_{1}}-\mu u_{1} \\
2 \frac{\varphi_{2}}{\varphi_{1}} & 0
\end{array}\right)
$$

(it is assumed hereafter that $\sigma$ is a scalar function of $\lambda$ ). The direct and dual ZS problems are covariant with respect to this EDT: matrix functions $\tilde{\Psi}$ and $\tilde{\Xi}$ are the solutions of Eqs.(10,11) with potential $\tilde{P}^{(0)}$. The second EDT of direct problem has a form:

$$
\begin{gathered}
\tilde{\Psi}=\sigma\left(\begin{array}{cc}
1 & -\frac{\varphi_{1}}{\varphi_{2}} \\
\frac{u_{2}}{2} & \lambda-\mu-\frac{u_{2} \varphi_{1}}{2 \varphi_{2}}
\end{array}\right) \Psi, \tilde{\Xi}=\frac{\Xi}{\sigma}\left(\begin{array}{cc}
\lambda-\mu-\frac{u_{2} \varphi_{1}}{2 \varphi_{2}} & \frac{\varphi_{1}}{\varphi_{2}} \\
-\frac{u_{2}}{2} & 1
\end{array}\right), \\
\tilde{P}^{(0)}=\left(\begin{array}{cc}
0 & -2 \frac{\varphi_{1}}{\varphi_{2}} \\
\frac{u_{2, x}}{2}-\frac{u_{2}^{2} \varphi_{1}}{2 \varphi_{2}}-\mu u_{2} & 0
\end{array}\right) .
\end{gathered}
$$

In similar manner one can define the EDT's of dual problem, which depend explicitly on a solution of the dual ZS problem (11). Successive carrying out of an EDT of direct problem and proper one of dual problem leads to so-called binary DT (BDT) 25, 26]:

$$
\begin{gathered}
\tilde{\Psi}=\sigma\left(E-\frac{\mu-\nu}{\lambda-\nu} R\right) \Psi, \quad \tilde{\Xi}=\frac{\Xi}{\sigma}\left(E-\frac{\mu-\nu}{\mu-\lambda} R\right), \\
\tilde{P}^{(0)}=P^{(0)}+(\mu-\nu)\left[P^{(1)}, R\right],
\end{gathered}
$$

where matrix $\mathrm{R}$ is the projector: $R_{i j}=\varphi_{i} \chi_{j} /(\chi, \varphi), \varphi=\left(\varphi_{1}, \varphi_{2}\right)^{T}$ is the vector solution of Eq.(10) with $\lambda=\mu, \chi=\left(\chi_{1}, \chi_{2}\right)$ is the vector solution of Eq.(11) with $\lambda=\nu$.

It is seen that the potential in the Lax pairs for $\mathrm{P}_{\mathrm{II}}$ is the symmetric matrix. The DT technique is convenient for keeping the reduction constraints imposed on the coefficients of the spectral problems [27]. We can obtain the transformed potentials to satisfy this reduction, performing the iterations of BDT [25, 26].

The DT's presented in this section allow us to build infinite hierarchy of solutions of nonlinear PDE's integrable in the frameworks of the IST method. It is supposed in so doing that vectors $\varphi$ and $\chi$ are the solutions of Lax pairs associated with given PDE. Unfortunately, this approach is unfit for obtaining the transformations, which generate infinite hierarchy of solutions of nonlinear ODE's representable as the compatibility condition of Lax pairs. Vectors $\varphi$ and $\chi$ cannot be regarded as the solutions of systems (1,2), since any transformation of the second equations of these Lax pairs ought to have no the kernel. The basic idea of the generalization of the DT technique for ODE's is to put the points, in which the DT of the first equations of corresponding Lax pairs has the kernel, into the singular points of the second equations of Lax pairs. For this aim we will assume in the next section that $\varphi$ and $\chi$ are the solutions of systems (1,2) in the points $\lambda=\mu$ and $\lambda=\nu$. Then we will consider the limits in the formulas of DT's, tending the points $\mu$ and $\nu$, in which the kernel exists, to singular point $\lambda=0$. 


\section{Transformations OF ODE's}

To fulfill the procedure suggested in the previous section we need the explicit expressions of the asymptotic expansions of solutions of Lax pairs (1,2) with coefficients given by Eqs.(5, 6) or Eqs.(8,9) at singular point neighborhood.

If $\alpha$ is unequal to half-integer, the asymptotic expansion of the Lax pairs solutions of $\mathrm{P}_{\mathrm{II}}$ at neighborhood of point $\lambda=0$ have the form

$$
\begin{gathered}
\Psi=\Psi_{0}(E+\lambda \rho+o(\lambda)) \Lambda, \\
\Xi=\Lambda^{-1}(E-\lambda \rho+o(\lambda)) \Psi_{0}^{-1} .
\end{gathered}
$$

Here we use notations

$$
\begin{gathered}
\Psi_{0}=\left(\begin{array}{cc}
d & d^{-1} \\
d & -d^{-1}
\end{array}\right), \Lambda=\left(\begin{array}{cc}
\lambda^{\alpha} & 0 \\
0 & \lambda^{-\alpha}
\end{array}\right), \rho=\left(\begin{array}{cc}
0 & \Delta_{1} \\
\Delta_{2} & 0
\end{array}\right), \\
\Delta_{1}=\frac{\left(2 u_{x}+2 u^{2}+x\right) d^{-2}}{2 \alpha-1}, \Delta_{2}=\frac{\left(2 u_{x}-2 u^{2}-x\right) d^{2}}{2 \alpha+1}, d=\exp \left(\int u d x\right) .
\end{gathered}
$$

The expansions in series of solutions of Lax pairs contain the logarithmic terms in the case of half-integer $\alpha$.

The asymptotic expansions of the solutions of Lax pairs of Eq.(7) at neighborhood of point $\lambda=0$ are given by Eqs.(18,19), in which the coefficients are defined as follows:

$$
\Psi_{0}=\left(\begin{array}{cc}
c_{1} & c_{2} \\
\frac{\alpha-z}{w z} c_{1} & -\frac{c_{2}}{w}
\end{array}\right), \Lambda=\left(\begin{array}{cc}
\mathrm{e}^{\left(-\frac{\alpha}{2 \lambda}+\frac{\gamma}{\alpha} \ln \lambda\right)} & 0 \\
0 & \mathrm{e}^{\left(\frac{\alpha}{2 \lambda}-\frac{\gamma}{\alpha} \ln \lambda\right)}
\end{array}\right)
$$

where functions $c_{1}$ and $c_{2}$ solve equations: $c_{1, x}=\frac{\alpha-z}{x} u c_{1}, c_{2, x}=-\frac{z}{x} u c_{2}$.

These asymptotic expansions allow us to apply formulas of the DT's for deriving the transformations of ODE's. Having the reduction constraint on potential $P^{(0)}$, we start from BDT (16, 17) in the case of $\mathrm{P}_{\mathrm{II}}$. To satisfy condition $\operatorname{Sp} Q=0$ under performing the transformation we put $\sigma=1$. Substituting

$$
\begin{gathered}
\varphi=\left.\Psi\right|_{\lambda=\mu}\left(\begin{array}{l}
a_{1} \\
a_{2}
\end{array}\right), \\
\chi=\left.\left(b_{1}, b_{2}\right) \Xi\right|_{\lambda=\nu}
\end{gathered}
$$

$\left(a_{1}, a_{2}, b_{1}, b_{2}\right.$ are constants) and considering the limits $\mu \rightarrow 0$ and $\nu \rightarrow 0$ in the BDT formulas, taking into account Eqs. 18, 19,20), we obtain two transformations of Lax pairs solutions and corresponding well-known transformations of $\mathrm{P}_{\text {II }}$ (e.g., see [15]):

$$
\left\{\begin{array}{l}
\tilde{\Psi}=\Psi+\ln _{x}\left(\Delta_{1}\right)\left(\sigma_{3}+i \sigma_{2}\right) \Psi /(2 \lambda) \\
\tilde{\Xi}=\Xi-\ln _{x}\left(\Delta_{1}\right)\left(\sigma_{3}+i \sigma_{2}\right) \Xi /(2 \lambda) \\
\tilde{u}=u+\ln _{x} \Delta_{1} \\
\tilde{\alpha}=1-\alpha
\end{array} \quad, \quad\left\{\begin{array}{l}
\tilde{\Psi}=\Psi+\ln _{x}\left(\Delta_{2}\right)\left(\sigma_{3}-i \sigma_{2}\right) \Psi /(2 \lambda) \\
\tilde{\Xi}=\Xi-\ln _{x}\left(\Delta_{2}\right)\left(\sigma_{3}-i \sigma_{2}\right) \Xi /(2 \lambda) \\
\tilde{u}=u-\ln _{x} \Delta_{2} \\
\tilde{\alpha}=-1-\alpha
\end{array} .\right.\right.
$$

The formulas of transformations coincide with presented above or follow from them as limits in the case of half-integer $\alpha$.

The transformations of Eq.(7) are constructed similarly to that of $\mathrm{P}_{\mathrm{II}}$. Substituting the asymptotic expansions (18,21) in formulas of EDT's (12,14) accordingly 
to Eq.(22) and considering limit $\mu \rightarrow 0$, we obtain four transformations of solutions and coefficients of Lax pairs:

$$
\begin{aligned}
& \left\{\begin{array}{l}
\tilde{\Psi}=\left(\begin{array}{cc}
\lambda+\frac{u_{1}}{2} \varepsilon_{1}^{ \pm} & -\frac{u_{1}}{2} \\
-\varepsilon_{1}^{ \pm} & 1
\end{array}\right) \frac{\Psi}{\sqrt{\lambda}}, \tilde{\Xi}=\frac{\Xi}{\sqrt{\lambda}}\left(\begin{array}{cc}
1 & \frac{u_{1}}{2} \\
\varepsilon_{1}^{ \pm} & \lambda+\frac{u_{1}}{2} \varepsilon_{1}^{ \pm}
\end{array}\right), \\
\tilde{\beta}=\beta+\frac{1}{2}, \tilde{v}_{1}=A_{1}^{ \pm}+\frac{v_{1}}{2 x}, \tilde{v}_{2}=2 x \varepsilon_{1}^{ \pm}, \quad \tilde{w}_{1}=\frac{v_{1}}{2 x}\left(2 q-\frac{v_{1} v_{2}}{2 x}+\right. \\
\left.+w_{1} \varepsilon_{1}^{ \pm}+A_{1}^{ \pm} \varepsilon_{1}^{ \pm}\right), \tilde{w}_{2}=v_{2}-2 \beta \varepsilon_{1}^{ \pm}-v_{1} \varepsilon_{1}^{ \pm 2}, \tilde{q}=q-\frac{v_{1} v_{2}}{2 x}+A_{1}^{ \pm} \varepsilon_{1}^{ \pm},
\end{array}\right. \\
& \left\{\begin{array}{l}
\tilde{\Psi}=\left(\begin{array}{cc}
\frac{u_{2}}{2} & \lambda-\frac{u_{2}}{2} \varepsilon_{2}^{ \pm}
\end{array}\right) \frac{\Psi}{\sqrt{\lambda}}, \tilde{\Xi}=\frac{\Xi}{\sqrt{\lambda}}\left(\begin{array}{cc}
\lambda-\frac{u_{2}}{2} \varepsilon_{2}^{ \pm} & \varepsilon_{2}^{ \pm} \\
-\frac{u_{2}}{2} & 1
\end{array}\right), \\
\tilde{\beta}=\beta-\frac{1}{2}, \quad \tilde{v}_{1}=-2 x \varepsilon_{2}^{ \pm}, \quad \tilde{v}_{2}=A_{2}^{ \pm}-\frac{v_{2}}{2 x}, \quad \tilde{w}_{1}=v_{1}+2 \beta \varepsilon_{2}^{ \pm}-v_{2} \varepsilon_{2}^{ \pm 2}, \\
\tilde{w}_{2}=-\frac{v_{2}}{2 x}\left(-2 q+\frac{v_{1} v_{2}}{2 x}+w_{2} \varepsilon_{2}^{ \pm}+A_{2}^{ \pm} \varepsilon_{2}^{ \pm}\right), \quad \tilde{q}=q-\frac{v_{1} v_{2}}{2 x}-A_{2}^{ \pm} \varepsilon_{2}^{ \pm},
\end{array}\right.
\end{aligned}
$$

where $\varepsilon_{1}^{ \pm}=\frac{1}{\varepsilon_{2}^{ \pm}}=-\frac{2 q \pm \alpha}{2 w_{1}}, A_{1}^{ \pm}=w_{1}+\frac{\beta}{x} v_{1}+\frac{v_{1}^{2}}{2 x} \varepsilon_{1}^{ \pm}, A_{2}^{ \pm}=w_{2}+\frac{\beta}{x} v_{2}-\frac{v_{2}^{2}}{2 x} \varepsilon_{2}^{ \pm}$.

(To keep condition $\operatorname{Sp} Q=0$ we assume $\sigma=\lambda^{-1 / 2}$ in the formulas of EDT's.)

These transformations lead to the following ones of solutions and parameters of Eq.(17) $\{u, \alpha, \beta, \gamma\} \stackrel{T_{i}}{\longrightarrow}\{\tilde{u}, \tilde{\alpha}, \tilde{\beta}, \tilde{\gamma}\}(i=1, \ldots, 4)$ :

$$
\begin{gathered}
T_{1}: \quad \tilde{u}=-\frac{2 x\left(x u_{x}+\alpha u^{2}-2 x-2(\beta+1) u\right)}{u\left(\alpha\left(x u_{x}+\alpha u^{2}-2 x\right)-2 \gamma u\right)}, \quad \tilde{\alpha}=\alpha, \quad \tilde{\beta}=\beta+\frac{1}{2}, \quad \tilde{\gamma}=\gamma-\frac{\alpha}{2} \\
T_{2}: \quad \tilde{u}=\frac{2 x\left(x u_{x}-\alpha u^{2}-2 x-2(\beta+1) u\right)}{u\left(\alpha\left(x u_{x}-\alpha u^{2}-2 x\right)+2 \gamma u\right)}, \quad \tilde{\alpha}=\alpha, \tilde{\beta}=\beta+\frac{1}{2}, \tilde{\gamma}=\gamma+\frac{\alpha}{2} \\
T_{3}: \tilde{u}=\frac{2 x\left(x u_{x}+\alpha u^{2}+2 x+2 \beta u\right)}{u\left(\alpha\left(x u_{x}+\alpha u^{2}+2 x\right)-2 \gamma u\right)}, \quad \tilde{\alpha}=\alpha, \quad \tilde{\beta}=\beta-\frac{1}{2}, \tilde{\gamma}=\gamma-\frac{\alpha}{2} \\
T_{4}: \quad \tilde{u}=-\frac{2 x\left(x u_{x}-\alpha u^{2}+2 x+2 \beta u\right)}{u\left(\alpha\left(x u_{x}-\alpha u^{2}+2 x\right)+2 \gamma u\right)}, \quad \tilde{\alpha}=\alpha, \quad \tilde{\beta}=\beta-\frac{1}{2}, \tilde{\gamma}=\gamma+\frac{\alpha}{2}
\end{gathered}
$$

The EDT's of dual pair give the same transformations. The transformations obtained commute and transformations $T_{4}$ and $T_{3}$ are inverse to $T_{1}$ and $T_{2}$ respectively. One can note that Eq.(7]) is invariant under changing $\alpha \rightarrow-\alpha$. This symmetry connects transformations $T_{1}$ and $T_{2}, T_{3}$ and $T_{4}$. It should be stressed that a form of these transformations differs from one considered in 15 .

If $\gamma= \pm \alpha \beta$ or $\gamma= \pm \alpha(\beta+1)$, particular solutions of Eq.(7) satisfy the Riccati equations. In these cases we obtain, performing the transformations of Eq.(17), which keep this condition, two transformations

$$
\left\{\begin{array}{l}
\tilde{\psi}=\sqrt{x}\left(\psi_{x}-\frac{\beta+1}{x} \psi\right) \\
\tilde{\beta}=\beta+\frac{1}{2}
\end{array},\left\{\begin{array}{l}
\tilde{\psi}=\sqrt{x}\left(\psi_{x}+\frac{\beta}{x} \psi\right) \\
\tilde{\beta}=\beta-\frac{1}{2}
\end{array}\right.\right.
$$

of the linearization of mentioned Riccati equations: $\psi_{x x}-\left(\frac{2 \alpha}{x}+\frac{\beta(\beta+1)}{x^{2}}\right) \psi=0$.

So, the modification of the DT technique presented here allows us to obtain the transformations of solutions of the nonlinear ODE's admitting the compatibility 
condition representation. At the same time we derive the Schlesinger transformations of associated Lax pairs. This approach can be applied for finding the transformations of nonlinear systems of ODE's and their Lax pairs that have different types of the singularities and the reduction constraints of the coefficients.

\section{REFERENCES}

[1] H. Flaschka: J. Math. Phys. 21, 1016-1018 (1980); H. Flaschka and A.C. Newell: Commun. Math. Phys. 76, 65-116 (1980).

[2] R. Fuchs: Math. Ann. 63, 301-321 (1906); R. Garnier: Ann. Ec. Norm. Sup. 29, 1-126 (1912).

[3] M. Jimbo and T. Miwa: Physica D2, 401-448 (1981).

[4] M.J. Ablowitz and H. Segur: Phys. Rev. Lett. 38, 1103-1106 (1977); M.J. Ablowitz, A. Ramani and H. Segur: Lett. Nuovo Cim. 23, 333-338 (1978); J. Math. Phys. 21, 715-721 (1980); J. Math. Phys. 21, 1006-1015 (1980).

[5] M.J. Ablowitz and P.A. Clarkson: Lect. Note Math. 149 (1991).

[6] M.D. Kruskal and P.A. Clarkson: Stud. Appl. Math. 86, 87-165 (1992).

[7] N.A. Lukashevich: Diff. Eqns. 7, 853-854 (1971).

[8] A.P. Vorob'ev: Diff. Eqns. 1, 58-59 (1965).

[9] M. Boiti and F. Pempinelli: Il Nuovo Cimento 51B, 70-78 (1979).

[10] V.I. Gromak: Diff. Urav. 9, 2082-2083 (1973) (in Russian).

[11] V.I. Gromak: Diff. Eqns. 11, 285-287 (1975).

[12] N.A. Lukashevich: Diff. Eqns. 3, 395-399 (1967).

[13] H. Airault: Stud. Appl. Math. 61, 31-53 (1979).

[14] N.A. Kudryashov and M.B. Soukharev: Phys. Lett. A237, 206-216 (1998).

[15] A.S. Fokas and M.J. Ablowitz: J. Math. Phys. 23, 2033-2042 (1982).

[16] P.A. Clarkson, N. Joshi and A. Pickering: Inverse Problems 15, 175-187 (1999).

[17] P.R. Gordoa, N. Joshi and A. Pickering: Nonlinearity 12, 955-968 (1999).

[18] L. Schlesinger: J. Reine Angewandte Math. 141, 96-145 (1912).

[19] M. Jimbo, T. Miwa and K. Ueno: 1981 Physica D2, 306-352 (1981); M. Jimbo and T. Miwa: Physica D4, 26-46 (1981).

[20] A.S. Fokas, U. Mugan and M.J. Ablowitz: Physica D30, 247-283 (1988), U. Mugan and A.S. Fokas: J. Math. Phys. 33, 2031-2055 (1992); U. Mugan and A. Sakka: J. Math. Phys. 36, 1284-1298 (1995).

[21] V.B. Matveev and M.A. Salle: Darboux Transformation and Solitons, Springer, Heidelberg 1991.

[22] H.C. Morris and R.K. Dodd: Phys. Lett. 75A, 249-253 (1980).

[23] S.B. Leble and N.V. Ustinov: J. Math. Phys. 34, 1421-1428 (1993).

[24] S.B. Leble and N.V. Ustinov: Deep Reductions for Matrix Lax Systems, Invariant Forms and Elementary Darboux Transforms, in Nonlinear Evolution Equations and Dynamical Systems VIII, V. Makhankov, I. Puzynin and O. Pashaev eds., World Scientific, Singapore 1993.

[25] S.B. Leble and N.V. Ustinov: Solitons of Nonlinear Equations Associated with Degenerate Spectral Problem of the Third Order, in Nonlinear Theory and its Applications (NOLTA'93), M. Tanaka and T. Saito eds, vol. 2, World Scientific, Singapore 1993.

[26] N.V. Ustinov: J. Math. Phys. 39, 976-985 (1998).

[27] S.B. Leble and N.V. Ustinov: J. Phys. A26, 5007-5016 (1993); Inverse Problems 10, 617-633 (1994).

Department of Theoretical Physics, Kaliningrad State University, Al. Nevsky street 14, 236041, Kaliningrad, Russia 